\title{
Efficiency of Power Generation by LNG Cold Energy
}

\author{
LIU Yanni GUO Kaihua* \\ School of Engineering \\ Sun Yat-Sen University \\ Guangzhou, China \\ guokaih@mail.sysu.edu.cn
}

\begin{abstract}
In this paper, a comparative study for the practical power generation from LNG cold energy with the Organic Rankine Cycle (ORC) + Direct Expansion (DE) combined cycle, the ordinary ORC and the irreversible Carnot cycle has been performed. The optimized LNG cold energy utilization temperature for obtaining the highest efficiency of power generation were revealed and the characteristics of the enthalpy and entropy as well as the availability for the LNG cold sinks with different pressures were studied. The results show that the optimized LNG utilization temperature exists and increases with the LNG vaporizing pressure, while the power output by unit LNG and the availability efficiency are reduced accordingly. For applying the higher LNG vaporizing pressure, the efficiency of the combined cycle is considerably superior to the ordinary Rankine cycle, due to the recovery of power from the direct expansion.
\end{abstract}

Key words-LNG; cold energy; power generation; ORC; efficiency

\section{INTRODUCTION}

Liquefied Natural Gas (LNG) is one of the most important world energy trading resources and has been widely used for industrial and civil life nowadays. LNG is produced by liquefaction of natural gas in which the water acid gases and other impurities have been removed. Therefore, LNG is clean and plays an important role in reducing pollution and protecting environment. LNG is necessary to be re-gasified before distributed to the users and it absorbs latent heat (about $800 \mathrm{~kJ} \cdot \mathrm{kg}-1$ ). This is usually called cold energy and taken away by seawater.

In the past decades, many efforts were dedicated to develop the ways of LNG cold energy utilization, such as power generation, air separation and so on. Power generation by LNG cold energy is the most significant one and has been applied in many large LNG receiving terminals worldwide. Researches on LNG cold power generation have been done a lot by some research institutions in China, such as Institute of Engineering Thermophysics, Chinese Academy of Science [1-4]. Organic Rankine Cycle (ORC), Direct Expansion (DE) cycle and Organic Rankine Cycle $(\mathrm{ORC})+$ Direct Expansion (DE) combined cycle are commonly used cycles[5]. Studies[6-9] were focused on selection of the working fluid, analysis of the parameters, optimization of the cycle performance, and so on. In this paper, the characteristics of the enthalpy and entropy as well as the availability for the LNG cold sinks with different pressures were studied and the performances of the practical power generation from LNG cold energy with the Organic Rankine Cycle (ORC) + Direct Expansion (DE) combined cycle, the ordinary ORC and the irreversible Carnot cycle has been analyzed. The optimized LNG cold energy utilization temperature for obtaining the highest efficiency of power generation was revealed.

\section{AVAILABILITY OF LNG AT DIFFERENT PRESSURES}

\section{A. Cold energy properties of $L N G$}

LNG vaporizing pressure differs with supply requirements. The pressures of $0.6 \mathrm{MPa}, 3.0 \mathrm{MPa}$ and 7.0 $\mathrm{MPa}$ are typical for steam power generation, local grids and long distance pipelines, relatively. The availability of LNG will vary with the vaporizing pressures. Fig. 1 and Fig. 2 show characteristics of the enthalpy and entropy of a kind of LNG (mole fraction: $\mathrm{N}_{2}=0.0007, \quad \mathrm{CH}_{4}=0.8877$, $\mathrm{C}_{2} \mathrm{H}_{6}=0.0754, \quad \mathrm{C}_{3} \mathrm{H}_{8}=0.0259, \quad \mathrm{nC}_{4} \mathrm{H}_{10}=0.0056, \quad \mathrm{iC}_{4} \mathrm{H}_{10}$ $\left.=0.0045, \mathrm{nC}_{5} \mathrm{H}_{12}=0.0001, \mathrm{iC}_{5} \mathrm{H}_{12}=0.0001\right)$ at $1 \mathrm{~atm}$ and three other typical pressures. At subcritical condition, there exists phase change, while at supercritical one $(7.0 \mathrm{MPa})$, no obvious phase change displays. The changes of enthalpy and entropy are close related with the vaporizing pressure.

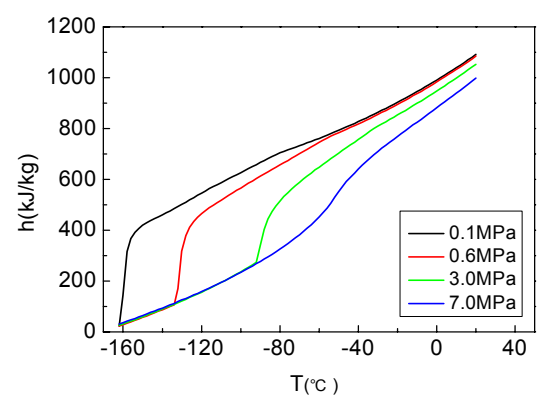

Fig.1 h-T diagram for LNG

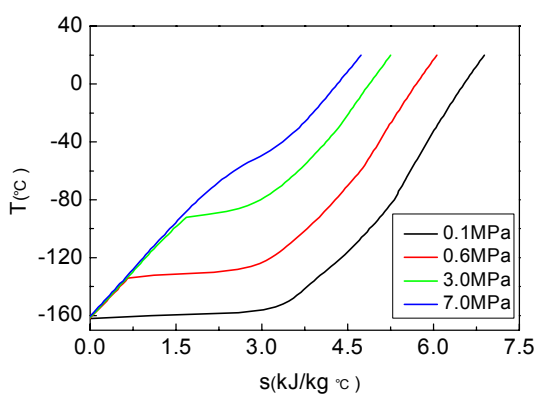

Fig. 2 T-s diagram for LNG

* Corresponding author: Tel: +86-20-39332893, Email: guokaih@mail.sysu.edu.cn. The work has been supported by the SYSU-BP LNG Center (No. 99103-9390001) and the Key Laboratory of LNG Cryogenic Technology, the Education Department of Guangdong Province (No. 39000-3211101). 


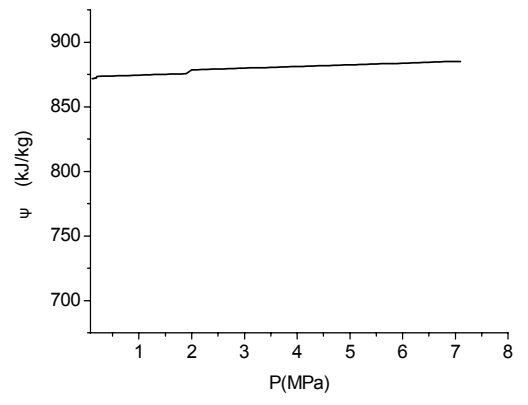

Fig. 3 Availability of LNG cold energy

When using seawater as the heat source and LNG as the heat sink, the temperature of heat sink will glide up due to heat exchange. The Carnot cycle can not be reversible in the heat releasing. To reduce the irreversibility, it is necessary to match the heat exchange process with the heat sink. Availability is the maximum reversible work that can be done by a system at given condition. According to the second law of thermodynamics, the availability of LNG cold energy can be calculated with Eq. (1) and is shown in Fig. 3.

$$
\psi=T_{0}\left(s_{0}-s\right)-\left(h_{0}-h\right)
$$

\section{B. Analysis of the irreversible Carnot cycle}
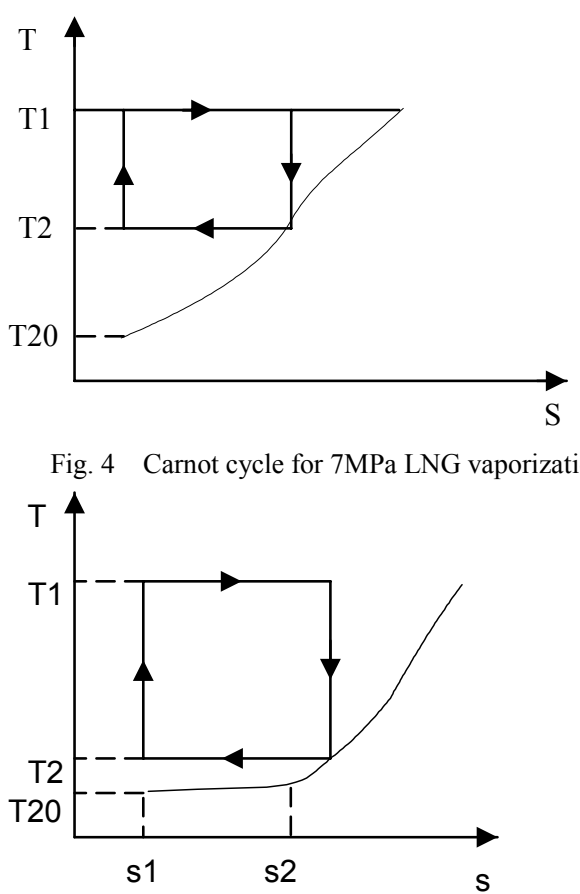

Fig. 5 Carnot cycle for 0.1MPa LNG vaporization

The two typical heat sinks: constant specific heat with variable temperature and constant temperature with phase change are shown in Fig. 4 and Fig. 5, respectively. The Carnot cycle analysis can show there a maximum unit power generation exists corresponding to an optimum $\mathrm{LNG}$ recover temperature. For the case of Fig. 4, corresponding to that of LNG vaporization at $7 \mathrm{MPa}$, the unit power generation, ${ }_{C}$, can be expressed as:

$$
W_{C}=Q_{2}\left(\frac{T_{1}}{T_{2}}-1\right)=C_{p}\left(T_{2}-T_{20}\right)\left(\frac{T_{1}}{T_{2}}-1\right)
$$

where $T_{1} 、 T_{20}$ are the temperatures before and after LNG vaporization, $T_{2}$ is the heat releasing temperature of the Carnot cycle, and $C_{p}$ is the average heat capacity of LNG. Then the optimized $T_{2}$ and the corresponding maximum unit power generation are:

$$
T_{2, \text { optimum }}=\sqrt{T_{1} T_{20}} \quad W_{C, \max }=C_{p}\left(\sqrt{T_{1}}-\sqrt{T_{20}}\right)^{2}
$$

For the case of Fig. 5, corresponding to that of LNG vaporization at $0.1 \mathrm{MPa}$, the unit power generation is:

$W_{C}=Q_{2}\left(\frac{T_{1}}{T_{2}}-1\right)=\left[T_{20} \frac{h_{f g}}{T_{p}}+C_{p}\left(T_{2}-T_{20}\right)\right]\left(\frac{T_{1}}{T_{2}}-1\right)$

The optimized $T_{2}$ and the corresponding maximum unit power generation then are:

$$
\begin{aligned}
& T_{2, \text { optimum }}=\sqrt{\left(1-\frac{h_{f g}}{T_{p} C_{p}}\right) T_{1} T_{20}} \\
& W_{C, \max }=\left(\sqrt{T_{20}\left(\frac{h_{f g}}{T_{p}}+C_{p}\right)}-\sqrt{C_{p} T_{1}}\right)^{2}
\end{aligned}
$$

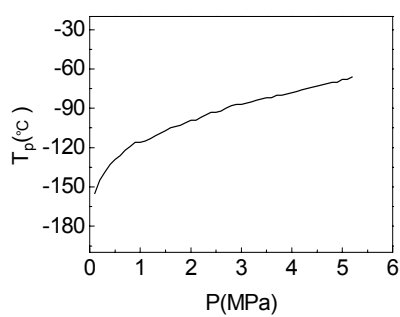

(a) Phase-change temperature

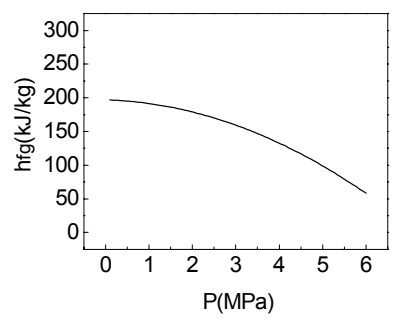

(b) Latent heat

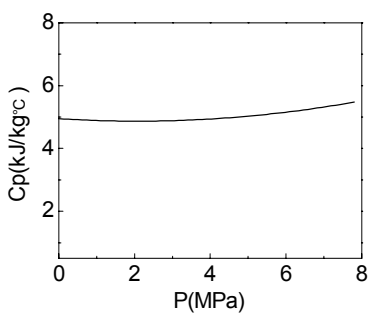

(c) Specific heat at constant pressure

Fig. 6 Equivalent thermodynamic properties of LNG at different pressures where $T_{p}, h_{f g}$ and $C_{p}$ are the equivalent phase-change 
temperature, latent heat and specific heat, respectively, at the given LNG pressure. The equivalent thermodynamic properties of LNG at different pressures are depicted in Fig. 6. With the equivalent properties for a given LNG vaporization pressure in Fig. 6, the optimum condition for LNG cold power generation can be found with Eq. (5).

\section{ORGANIC RANKINE CYCLE+DIRECT EXPANSION CYCLE}

In order to fully utilize of cryogenic and pressure energy, Organic Rankine cycle (ORC)+Direct Expansion (DE) combined cycle is suitable when LNG vaporization pressure is higher, e.g. $3 \mathrm{MPa}$ or $7 \mathrm{MPa}$, Fig. 7 shows the combined cycle schematically. The red part, which consists of a turbine, a heat exchanger (HX1), a pump and a heat exchanger (HX2), is the ORC, with propane as the working fluid.

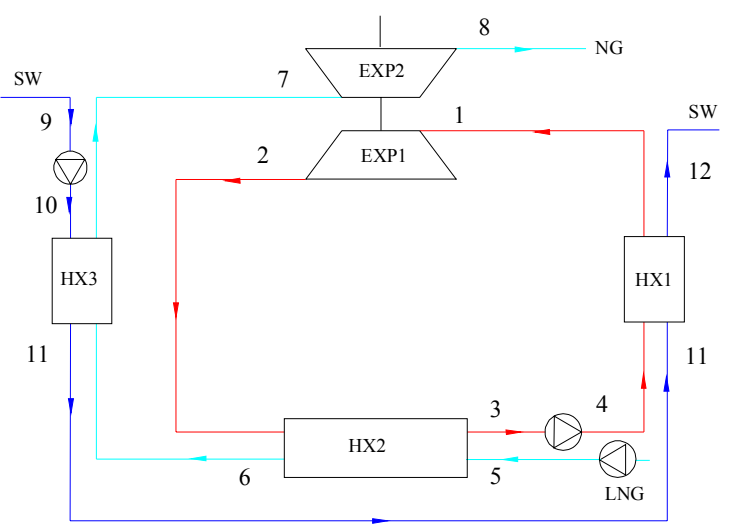

Fig. 7 ORC+DE combined cycle

Red: Propane Blue: Sea water Green: LNG

The operation parameters are: the sea water temperature $\mathrm{TW}=20^{\circ} \mathrm{C}$, the ambient pressure $\mathrm{P} 0=0.1 \mathrm{MPa}$, the efficiency of gas turbine $\eta_{\text {turbine }}=0.8$, efficiency of pump $\eta_{\text {pump }}=0.7$. The performance indicators are the unit LNG power generation and the availability efficiency, which are expressed as.

$$
w=\frac{\sum W_{\text {Tuibine }}-\sum W_{\text {Pump }}}{m_{L N G}} \quad \eta=\frac{w}{\psi}
$$

Fig. 8 and Fig. 9 show the variation of unit power generation and the availability efficiency with the utilization temperature of LNG at different pressures for the three cycles, named Carnot, Combined and Rankine, respectively. It can be seen that there is an optimum LNG utilization temperature to have the maximum unit power output. The optimum LNG utilization temperature increases with the LNG pressure, whereas unit power generation and availability efficiency decrease accordingly. At a constant LNG utilization temperature, the performance of the combined cycle is superior to the ordinary Rankine cycle but inferior to the Carnot cycle.

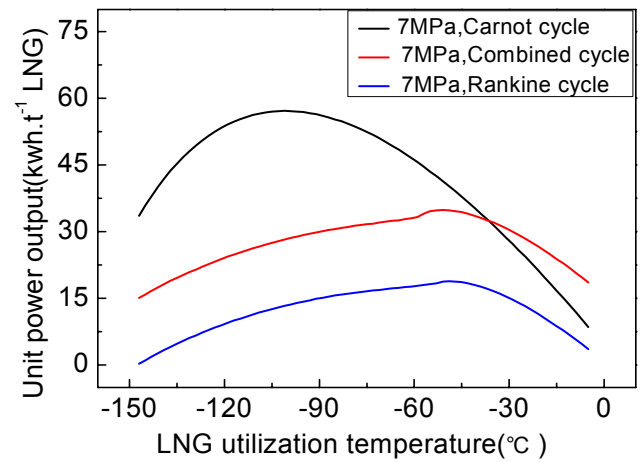

(a) LNG vaporizing pressure at $7 \mathrm{MPa}$

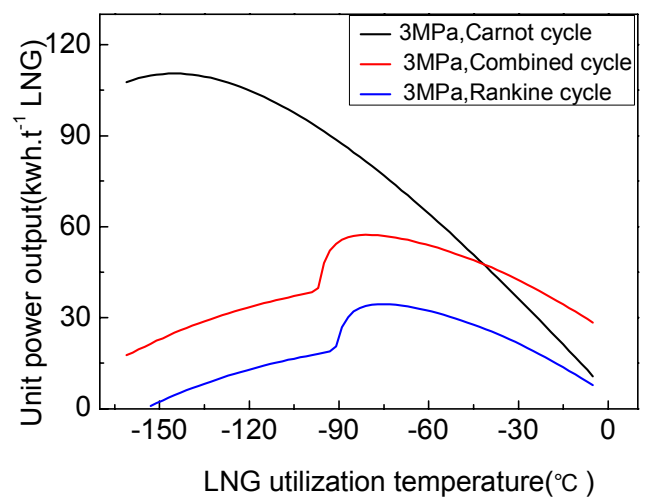

(b) $\mathrm{LNG}$ vaporizing pressure at $3 \mathrm{MPa}$

Fig. 8 Variation of the unit power output with LNG utilization temperature for different cycles

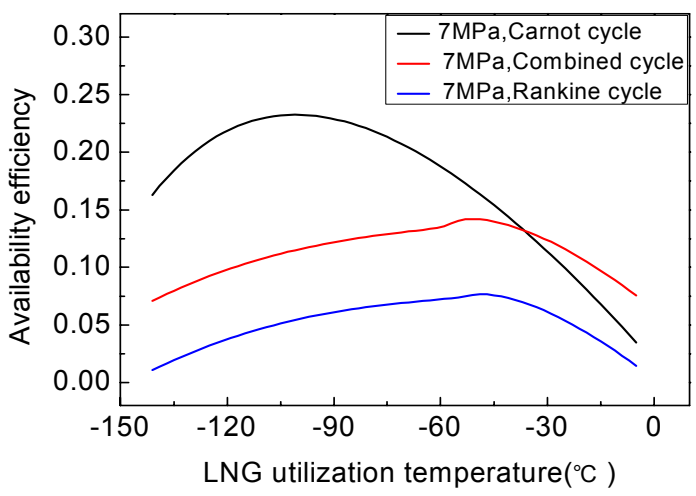

(a) LNG vaporizing at $7 \mathrm{MPa}$

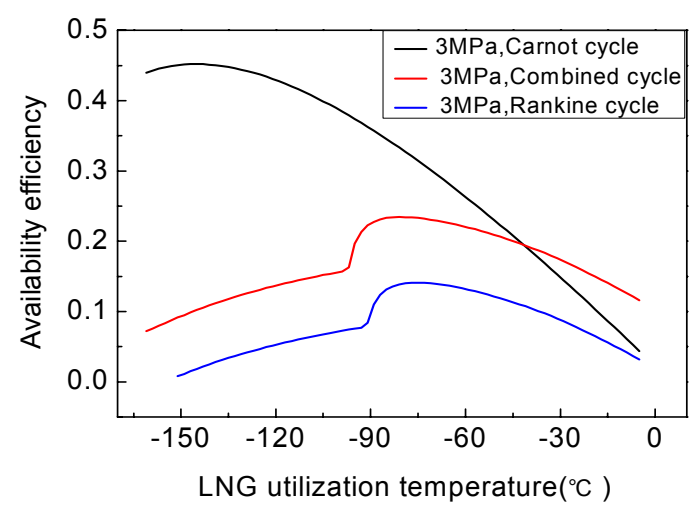

(b) $\mathrm{LNG}$ vaporizing at $3 \mathrm{MPa}$

Fig.9 Variation of availability efficiency with LNG utilization temperature for different cycles 


\section{COMPARISON BETWEEN ORC AND THE COMBINED CYCLE}

When LNG vaporization pressure is low, such as 0.6 $\mathrm{MPa}$, it's not practical to apply the direct expansion cycle. For the simple ORC, the LNG pressure can be lower. Fig. 10 and Fig. 11 show the variation of unit power and availability efficiency with the LNG utilization temperature to lower LNG pressures for ORC. When LNG vaporization pressure is $0.6 \mathrm{MPa}$, the optimum LNG utilization temperature is $-120^{\circ} \mathrm{C}$, unit power generation and availability efficiency increases by $80 \%$ compare with $3 \mathrm{MPa}$.

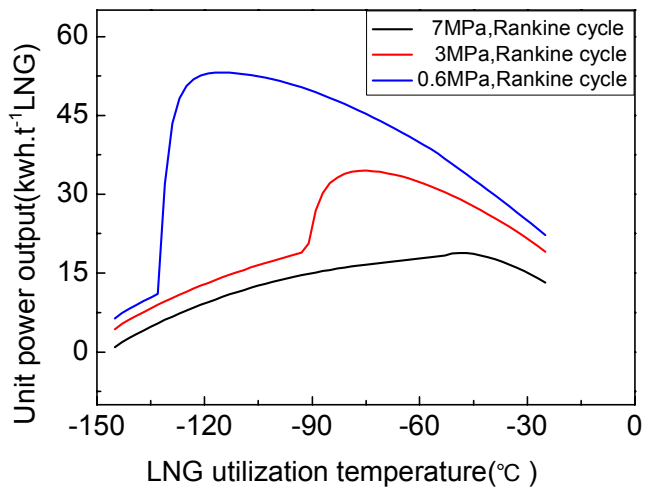

Fig. 10 Variation of unit power output with LNG utilization temperature for Rankine cycles

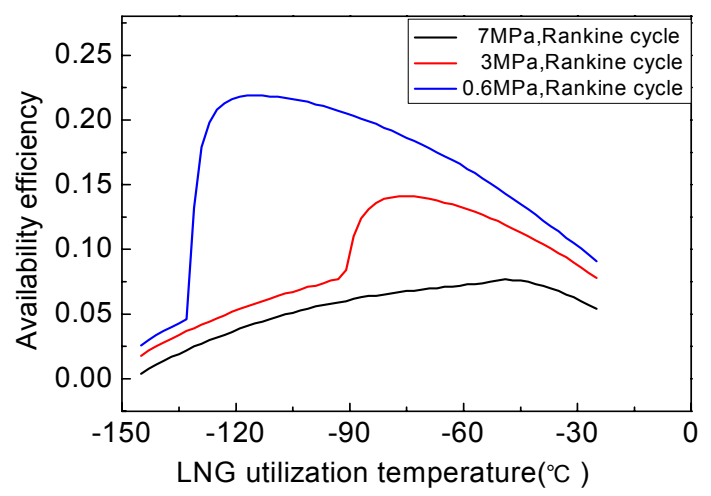

Fig. 11 Variation of availability efficiency with LNG utilization temperature for Rankine cycles

For comparison the performances of the combined ORC+DE cycle and the simple ORC cycle, the optimum condition and the maximum power output and efficiency for different LNG vaporization pressure (heat sink) are listed in Table 1. For both cycles, the optimum LNG utilization temperature increases with the LNG pressure, while the unit power generation and availability efficiency decrease significantly. Therefore, lowering the LNG vaporization pressure will be advantageous to power generation if the distributed pressure to the grid is allowable. It can be seen that the maximum unit power generation of the combined cycle is 1.97 and 1.66 times of that of the Rankine cycle for $7 \mathrm{MPa}$ and $3 \mathrm{MPa}$ of the $\mathrm{LNG}$ vaporization pressure. It means that the combined cycle is superior to Rankine cycle when the LNG vaporization pressure is higher.
TABLE 1 COMPARISON BETWEEN THE COMBINED AND RANKINE CYCLES

\begin{tabular}{|c|c|c|c|c|}
\hline Cycles & $\begin{array}{c}\text { Pressure / } \\
\text { MPa }\end{array}$ & $\begin{array}{c}\text { Optimized } \\
\text { LNG recover } \\
\text { temperature } \\
/{ }^{\circ} \mathrm{C}\end{array}$ & $\begin{array}{c}\text { Max unit } \\
\text { power } \\
\text { generation / } \\
\text { kwh.t }^{-1}\end{array}$ & $\begin{array}{c}\text { Max } \\
\text { availability } \\
\text { efficiency } \\
\text { \% }\end{array}$ \\
\hline Combined & 7 & -51 & 34.9 & 14.2 \\
& 3 & -81 & 57.3 & 23.4 \\
\hline Rankine & 7 & -49 & 18.8 & 7.7 \\
& 3 & -75 & 34.5 & 14.1 \\
& 0.6 & -115 & 53.2 & 21.9 \\
\hline
\end{tabular}

\section{CONCLUSION}

The optimized LNG cold energy utilization temperature for obtaining the highest efficiency of power generation were revealed and the characteristics of the enthalpy and entropy as well as the availability for the LNG cold sinks with different pressures were studied. The analysis of the irreversible Carnot cycle was given. The optimum LNG recover temperature, the maximum unit power generation and availability efficiency for the three studied cycles can be concluded as the following:

For practical Organic Rankine cycle + Direct Expansion combined cycle, the variation of performance parameters has the similar characteristics with the irreversible Carnot cycle and the simple ORC. There exists an optimum LNG recover temperature, which increases with the LNG vaporization pressure, to lead the maximum unit power output and availability efficiency. For different LNG vaporization pressures, the optimum performance of the considered cycles will vary significantly.

When LNG vaporization pressure is higher, because of the recovery of direct expansion power, the performance of the combined $\mathrm{ORC}+\mathrm{DE}$ cycle is superior to the ordinary ORC.

\section{REFERENCE}

[1] Cheng Wenlong, ITO Takehiro, and Chen Zeshao, "A cryogenic power generation cycle for recovering cold Energy of LNG", Journal of China University of Science and Technology, 1999, vol. 29, pp. 671-676.

[2] Gao Lin, Wang Yu, Jin Hongguang, Liu Zelong, and Cai Ruixian, "A novel binary cycle with integration of low-level waste heat recovery and LNG cold energy utilization", Journal of Engineering Thermophysics , 2002, vol. 23, pp. 397-400.

[3] He Hongming, and Lin Wensheng, "Power generation using LNG cold energy", Cryogenics and Superconductivity, 2006, vol. 34, pp. 432-436.

[4] Wang Qiang, Li Yanzhong, and Chen Xi, "A cryogenic power system for recovering LNG (Liquefied Natural Gas) cooling energy based on a low-grade heat source", Journal of Engineering for Thermal Energy and Power, 2003, vol. 18, pp. 245-247.

[5] T. C. Hung, T. Y. Shai, and S. K. Wang, "A Review of Organic Rankine Cycles (ORCs) for the Recovery of Low-Grade Waste Heat", . Energy, 1997, vol. 22, pp. 661-667.

[6] H.D. Madhawa Hettiarachchi, Mihajlo Golubovic, William M. Woreka, and Yasuyuki Ikegami, "Optimum design criteria for an Organic Rankine cycle using low-temperature geothermal heat sources", Energy, 2007, vol. 32, pp. 1698-1706.

[7] X.D. Wang, and L. Zhao, "Analysis of zeotropic mixtures used in low-temperature solar Rankine cycles for power generation", Solar Energy, 2009, vol. 83, pp. 605-613.

[8] T. Miyazaki, Y.T. Kang, A. Akisawa, and T. Kashiwagi, "A combined power cycle using refuse incineration and LNG cold energy", Energy, 2000, vol. 25, pp. 639-655.

[9] Zheng Hao, Tang Ked, Jin Tao, Wang Jinbo, Xu Lijun, and Xiang Jingqi, "Advances in working fluids for Organic Rankine cycle", Energy Engineering, 2008, vol. 4, pp. 5-11. 